\title{
An Update on the Epidemiological Features of Imported Schistosomiasis and Cystic Echinococcosis in Kuwait, 2010-2018
}

\author{
Mohammad Al-Awadhi Jamshaid lqbal Suhail Ahmad \\ Department of Microbiology, Faculty of Medicine, Kuwait University, Safat, Kuwait
}

\section{Highlights of the Study}

- The recorded rates of seroprevalence of schistosomiasis and cystic echinococcosis (CE) in Kuwait were 19.0 and $5.8 \%$, respectively, during the period 2010-2018.

- All schistosomiasis cases were imported, and a significant majority of CE cases were detected among non-Kuwaiti immigrant workers.

- Implementation of preventive measures in endemic countries and Kuwait has resulted in declining trends of both parasitic infections in Kuwait.

\section{Keywords}

Schistosomiasis · Echinococcosis · Hydatid · Prevalence · Seroepidemiology

\begin{abstract}
Objective: Kuwait is considered a non-endemic country for most parasitic infections. However, $\sim 70 \%$ of 4.7 million residents in Kuwait are expatriates from Asian and African countries, which are endemic for parasitic infections. Results of microbiological investigations for schistosomiasis and cystic echinococcosis (CE) performed in a reference national laboratory were retrospectively collected and analyzed to provide an insight on the epidemiology of these 2 neglected tropical diseases in Kuwait. Subjects and Methods: Schistosoma infection in fecal and urine specimens from suspected patients was detected by microscopy. Schistosoma and CE
\end{abstract}

infections were also detected by indirect hemagglutination assays (IHAs) using blood specimens. Patients' epidemiological data were extracted from the laboratory records. $\boldsymbol{R} \boldsymbol{e}$ sults: The overall prevalence rates of schistosomiasis and CE were 19.0 and $5.8 \%$, respectively. Almost all schistosomiasis cases were seen among Egyptians, especially among males, and a significantly higher prevalence $(p<0.05)$ was seen for CE cases among the Syrian residents. A decreasing annual trend was observed for both the parasitic infections over time in Kuwait. Conclusions: This study confirmed that schistosomiasis is not autochthonous in Kuwait, as all cases were detected among expatriates from Schistosoma-endemic countries. Our data also showed that CE remains endemic among humans and livestock in Kuwait as is also seen in other Middle Eastern countries.

(c) 2021 The Author(s) Published by S. Karger AG, Basel karger@karger.com www.karger.com/mpp

Karger $\stackrel{\text { ' }}{5}$

GOPEN ACCESS
C 2021 The Author(s)

Published by S. Karger AG, Basel

This is an Open Access article licensed under the Creative Commons Attribution-NonCommercial-4.0 International License (CC BY-NC) (http://www.karger.com/Services/OpenAccessLicense), applicable to the online version of the article only. Usage and distribution for commercial purposes requires written permission.
Correspondence to:

Mohammad Al-Awadhi, paramo1983@hotmail.com 


\section{Introduction}

Kuwait is a small Arabian Gulf country in the Middle East. It borders Iraq in the north/northwest and Saudi Arabia in the south/southwest. Kuwait has an arid desert climate with little or no freshwater reservoirs, has mostly dry environment with mild winters and hot/dry summers with temperatures ranging between $45-53^{\circ} \mathrm{C}$. This highincome oil-producing country has experienced rapid socioeconomic development in recent years and has employed a large number of expatriate workers. The total population of $\sim 4.7$ million in 2019 included $70 \%$ expatriates and $30 \%$ Kuwaiti nationals. The expatriate population is dynamic and diverse, but mostly comes from India (22\%), Egypt (15\%), Bangladesh (6\%), the Philippines (5\%), Syria (3\%), Saudi Arabia (3\%), Pakistan (2\%), Sri Lanka (2\%), Nepal (1\%), Ethiopia (1\%), Jordan $(<1 \%)$, and Lebanon $(<1 \%)$ [1]. Many expatriates originate from countries endemic for parasitic and other infectious diseases [2-5].

In 2017, the WHO categorized schistosomiasis and cystic echinococcosis (CE), which cause significant human morbidity and mortality, as neglected tropical diseases $[6,7]$. Schistosoma spp. are waterborne parasites that infect more than 140 million people with an annual disease burden of 1.9 million disability-adjusted life years (DALYs). The infection is transmitted to humans when cercarial larvae released by the intermediate host penetrate the skin during bathing, swimming, or wading in contaminated water. Nearly 800 million people are at risk of acquiring the infection with $85 \%$ cases occurring in 42 sub-Saharan African countries [7,8]. The prevalence rates of $\sim 50 \%$ have been recorded in highly endemic communities where Schistosoma mansoni and S. haematobium are more common [7-9]. Egypt and other countries along the Nile River are ancient and major foci of schistosomiasis. Although autochthonous cases of schistosomiasis have never been detected in Kuwait due to lack of natural bodies of freshwater and the intermediate host, imported schistosomiasis cases have been reported, mainly among Egyptian expatriates [9].

Nearly 1 million people worldwide are affected by CE with an assessed disease burden of 183,573 DALYs [6]. The endemicity of CE depends on the presence of a large number of nomadic or semi-nomadic intermediate hosts including sheep and goat flocks, and their close contact with wild or domesticated canids (definitive host), which help in the transmission of infection to humans. CE is endemic in the WHO Eastern Mediterranean Region (EMR), which includes Kuwait [6]. The EMR is one of the most ancient foci of the domestic cycle of CE involving humans and domesticated animals including cattle, sheep, goats and stray dogs [10].

The major pathological effects of both schistosomiasis and CE are generally seen in patients with chronic infections. Chronic schistosomiasis may cause colonic polyposis, hepatic disorders/failure (by S. mansoni or S. japonicum), or bladder polyps/obstructive uropathies (by $S$. haematobium). In chronic $\mathrm{CE}$, the enlarged cysts exert pressure on surrounding organs causing many pathological changes by compression or obstruction. Since the prevalence of schistosomiasis and CE infection in Kuwait has not been determined in recent years, this study aimed to provide an update on the prevalence and other epidemiological features of these 2 parasitic infections.

\section{Subjects and Methods}

Extraction, Categorization, and Entry of Epidemiological Data

This laboratory-based epidemiological study was undertaken at the Parasitology Reference Laboratory (PRL), Department of Microbiology, Faculty of Medicine, Kuwait University. PRL is the only parasite reference laboratory in the country, and the biological specimens from all cases suspected of schistosomiasis or CE are referred to PRL for investigations. Patients' records referred for schistosomiasis and CE investigation from January 2010 to December 2018 were reviewed. The epidemiological data were retrieved from PRL registry logbooks and included: date of sample collection, age, gender, and nationality of the patient and the referral hospital. Patients with incomplete information were excluded from the study. The "non-Kuwaiti subjects of unknown nationality" group included stateless illegal residents, also known as the "Bidoon" (Arabic word meaning "without", i.e., without nationality) in Kuwait.

\section{Diagnostic Testing for Schistosomiasis}

The biological specimens from all cases suspected of schistosomiasis in Kuwait were referred to PRL for confirmation of infection. Fecal specimens were collected and concentrated via Fecal Parasites Concentrator kit (LTA S.r.l., Milano, Italy). Urine specimens were centrifuged for $5 \mathrm{~min}$ at $4,000 \mathrm{rpm}$ and the sediment was collected. Processed urine and stool specimens were examined by light microscopy for ova of Schistosoma spp. Blood specimens were examined by indirect hemagglutination assay (IHA) kit (Cellognost Schistosomiasis $H ®$, Siemens, Erlangen, Germany) for anti-S. mansoni IgG antibodies used according to manufacturer's instructions. Briefly, $175 \mu \mathrm{L}$ of [PBS, $\mathrm{pH}$ 8.0] were dispensed into the wells of column A of a microtiter plate (A1 to H1) and $50 \mu \mathrm{L}$ of PBS into the remaining wells except A12. Subsequently, $25 \mu \mathrm{L}$ of positive control serum were added to the buffer in A1, while 50 $\mu \mathrm{L}$ of diluted negative control were added to A12. Twenty-five microliters of test sera were added to wells B1 through H1. Subsequently, $50 \mu \mathrm{L}$ from well 1 (A1 to $\mathrm{H} 1$ ) were added to well 2 (B2 to $\mathrm{H} 2$ ), mixed and serially diluted from row A1 to A11 and finally, 50 $\mu \mathrm{L}$ were discarded from the last well. Cellognost ${ }^{\circledR}$ Schistosomiasis $\mathrm{H}$ Reagent $(25 \mu \mathrm{L})$ was added into the wells of rows $2-12$, the mi- 
Table 1. Epidemiological data of subjects referred for schistosomiasis investigation in Kuwait (2010-2018)

\begin{tabular}{|c|c|c|c|c|}
\hline Variable & $\begin{array}{l}\text { Total } \\
(n=977)\end{array}$ & $\begin{array}{l}\text { Negative } \\
(n=791)\end{array}$ & $\begin{array}{l}\text { Schistosomiasis cases } \\
(n=186)\end{array}$ & $p$ value \\
\hline $\begin{array}{l}\text { Age mean, years } \\
\text { Gender, } n(\%)\end{array}$ & 43.1 (95\% CI: 42.1, 44.1) & 43.2 (95\% CI: $42.05,44.4)$ & 42.6 (95\% CI: 40.5, 44.7) & 0.618 \\
\hline $\begin{array}{l}\text { Male }^{\mathrm{a}} \\
\text { Female }\end{array}$ & $\begin{array}{l}843(86.3) \\
134(13.7)\end{array}$ & $\begin{array}{l}674(80.0) \\
117(87.3)\end{array}$ & $\begin{array}{c}169(20.0)^{\mathrm{a}} \\
17(12.7)\end{array}$ & 0.044 \\
\hline \multicolumn{5}{|c|}{ Country of origin, $n(\%)$} \\
\hline $\begin{array}{l}\text { Egypt }^{\mathrm{a}} \\
\text { Non-Kuwaiti } \\
\text { Kuwait } \\
\text { Iraq } \\
\text { Others }{ }^{\mathrm{b}}\end{array}$ & $\begin{array}{c}531(54.4) \\
340(34.8) \\
61(6.2) \\
1(0.1) \\
44(4.5)\end{array}$ & $\begin{array}{l}402(75.7) \\
284(83.5) \\
61(100.0) \\
0 \\
44(100.0)\end{array}$ & $\begin{array}{l}129(24.3)^{\mathrm{a}} \\
56(16.5) \\
0 \\
1(100.0) \\
0\end{array}$ & $<0.001$ \\
\hline
\end{tabular}

a Indicates points of significance $(p<0.05) .{ }^{\mathrm{b}}$ Others: Syria $(n=15)$, India $(n=7)$, Sudan $(n=6)$, Saudi Arabia $(n=5)$, Bangladesh $(n=4), 2$ each from Ethiopia and Yemen, 1 each from Jordan, Eritrea, and Oman.

crotiter plate was placed on a shaker for $20 \mathrm{~s}$, covered with aluminum foil and incubated at room temperature for $2 \mathrm{~h}$. The results were evaluated with a cutoff titer of $1: 128$. Titers $\geq 1: 128$ were considered as positive cases.

\section{Diagnostic Testing for CE}

Patients with suspected CE, either clinically or on CT imaging, were referred to PRL for investigation of serological evidence of CE. Serological screening was performed by IHA kit (CellognostEchinococcosis ${ }^{\circledR}$ Kit, Siemens Healthcare Diagnostics Products $\mathrm{GmbH}$, Marburg, Germany) used according to the manufacturer's instructions and as described above except that Cellognost ${ }^{\circledR}$ Echinococcosis Reagent was used in place of Cellognost ${ }^{\circledR}$ Schistosomiasis H Reagent. The IHA results were evaluated with a cut-off titer of 1:128. Titers $\geq 1: 128$ were considered as positive cases.

\section{Statistical Analysis}

Pearson's $\chi^{2}$ was used to test association of infection with patient's gender, nationality, location, and year and month of sample collection. Student's $t$-test was used to measure the difference in age between infected and noninfected patients. A $p$ value $<0.05$ was considered as statistically significant. Statistical analyses were performed by using WinPepi software v11.65 (PEPI for Windows, Microsoft Inc., Redmond, WA, USA).

\section{Ethical Clearance}

Due to the retrospective nature of this study, ethical clearance was not required as the extracted data did not include any personal information and the identity of subjects remained anonymous.

\section{Results}

\section{Schistosomiasis}

A total of 977 subjects were referred from different hospitals in Kuwait and 186 (19.0\%) cases tested sero- logically positive for schistosomiasis (Table 1). In addition, 82 urine specimens were also screened microscopically for schistosomiasis, of which only 1 case was positive for S. haematobium ova. The age of referred subjects ranged from 1 to 84 years with a mean of 43.1 years (95\% CI: 42.1, 44.1). There was no difference in mean age between schistosomiasis cases [42.6 years (95\% CI: 40.5, 44.7)] and noninfected subjects [43.2 years (95\% CI: 42.0, 44.4)] ( $p=0.610)$. A significant majority of schistosomiasis cases were males $(n=169 ; 90.9 \%)(p=0.043)$. By country of origin, all schistosomiasis cases $(n=186)$ were non-Kuwaiti subjects with Egyptians as the dominant group ( $n=129$; $69.4 \%$ ) in comparison with other non-Kuwaiti nationalities $(p<0.001)$. The number of schistosomiasis cases were significantly higher in $2010(n=50)$ and 2,011 $(n=59)$, but thereafter consistently declined from $2012(n=25)$ to $2018(n=3)(p<0.001)$ (Fig. 1). In addition, the seropositivity and the estimated incidence rate per 100,000 persons showed significant and progressive decline for schistosomiasis during the study period.

Although schistosomiasis cases did not show significant monthly or seasonal bias ( $p=0.797$ ) (Fig. 2), twothirds of schistosomiasis cases $(n=122 ; 66.8 \%)$ were diagnosed during the winter and spring (December to May) seasons. Significantly, higher number of schistosomiasis cases were detected among subjects referred from Amiri Hospital $(n=67)$ and Adan Hospital $(n=$ $33)$ in comparison with other hospitals $(p=0.015)$ (Fig. 3). 
Fig. 1. Annual number of reported schistosomiasis and CE cases in Kuwait (20102018). CE, cystic echinococcosis.

Fig. 2. Monthly number of reported schistosomiasis and CE cases in Kuwait (20102018). CE, cystic echinococcosis.

Fig. 3. The number and percentage of schistosomiasis (left) and CE (right) cases referred from different hospitals in Kuwait (2010-2018). CE, cystic echinococcosis.
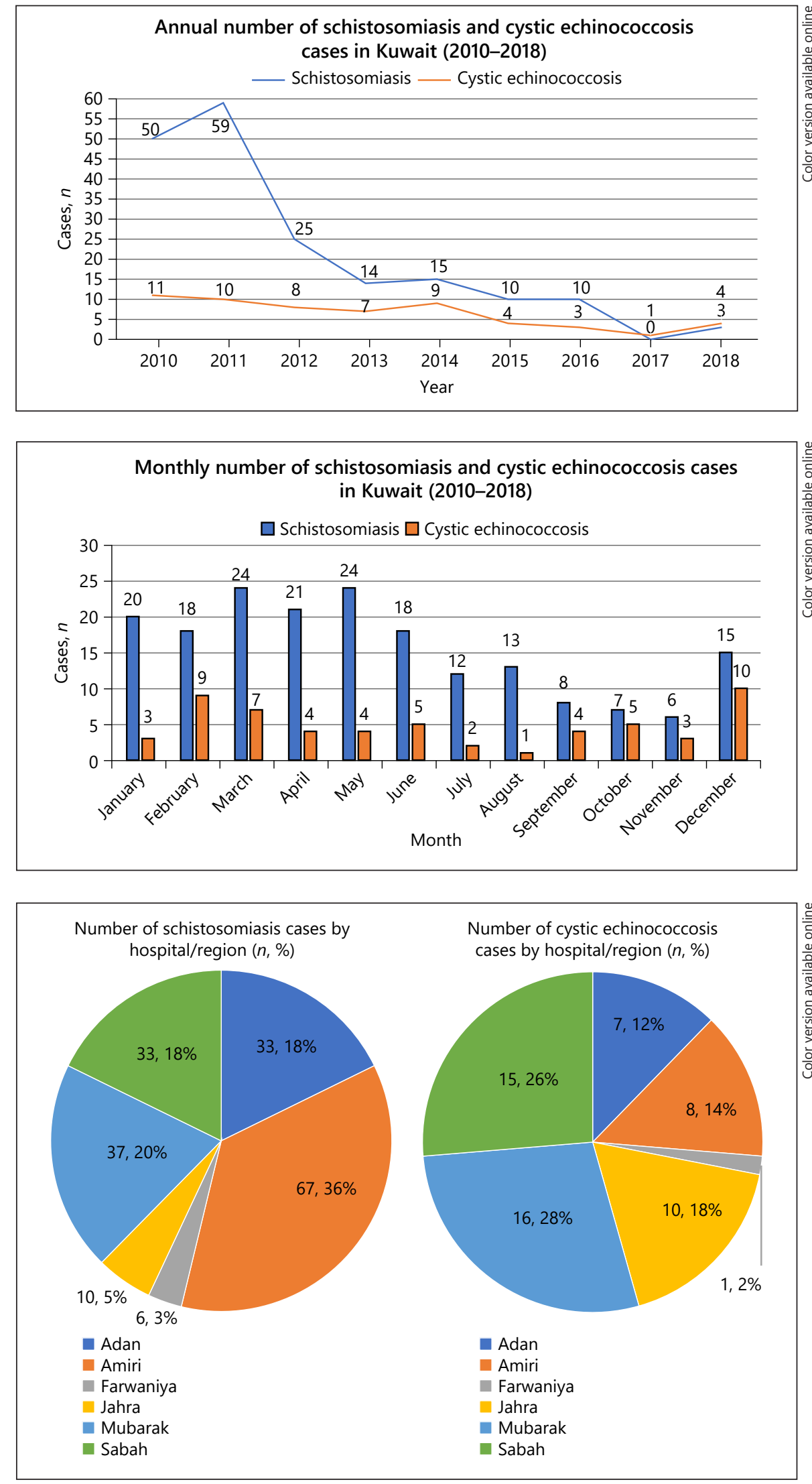
Table 2. Epidemiological data of subjects referred for CE investigation in Kuwait (2010-2018)

\begin{tabular}{|c|c|c|c|c|}
\hline Variable & Total $(n=991)$ & Negative $(n=934)$ & CE cases $(n=57)$ & $p$ value \\
\hline Age mean, years & 48.0 (95\% CI: 46.8, 49.2) & 48.2 (95\% CI: 47.0, 49.5) & 44.0 (95\% CI: 39.4, 48.6) & 0.117 \\
\hline \multicolumn{5}{|l|}{ Gender, $n(\%)$} \\
\hline Male & $517(52.2)$ & $484(93.6)$ & $33(6.4)$ & \multirow{2}{*}{0.373} \\
\hline Female & $474(47.8)$ & $450(94.9)$ & $24(5.1)$ & \\
\hline \multicolumn{5}{|c|}{ Country of origin, $n(\%)$} \\
\hline Kuwait & $423(42.7)$ & $408(96.5)$ & $15(3.5)$ & \\
\hline Non-Kuwaiti & $386(39.0)$ & $365(94.6)$ & $21(5.4)$ & \\
\hline India & $45(4.5)$ & $43(95.6)$ & $2(4.4)$ & \\
\hline Syria $^{a}$ & $30(3.0)$ & $20(66.7)$ & $10(33.3)^{\mathrm{a}}$ & \\
\hline Egypt & $28(2.8)$ & $26(92.9)$ & $2(7.1)$ & \\
\hline Lebanon & $12(1.2)$ & $9(75.0)$ & $3(25.0)$ & $<0.001$ \\
\hline Morocco & $3(0.3)$ & $2(66.7)$ & $1(33.3)$ & \\
\hline Afghanistan & $1(0.1)$ & 0 & $1(100.0)$ & \\
\hline Sudan & $1(0.1)$ & 0 & $1(100.0)$ & \\
\hline Tunisia & $1(0.1)$ & 0 & $1(100.0)$ & \\
\hline Others ${ }^{b}$ & $61(6.2)$ & $61(100.0)$ & 0 & \\
\hline
\end{tabular}

CE, cystic echinococcosis. ${ }^{\mathrm{a}}$ Indicates points of significance $(p<0.05) .{ }^{\mathrm{b}}$ Others: Saudi Arabia $(n=14)$, Philippines $(n=13)$, Bangladesh $(n=10)$, Pakistan $(n=5)$, 3 each from Iran, Jordan, Yemen, 2 each from Ethiopia and Sri Lanka, 1 each from Indonesia, Italy, Luxembourg, Malaysia, Palestine, and the UK.

\section{Cystic Echinococcosis}

A total of 991 subjects were referred from different hospitals in Kuwait to PRL for CE investigation and 57 (5.8\%) tested positive (Table 2). The age of subjects ranged from $<1$ to 97 years with a mean of 48.0 years. There was no significant difference in mean age between $\mathrm{CE}$ cases (44.0 years [95\% CI: 39.4, 48.6]) and noninfected subjects (48.2 years [95\% CI: 47.0, 49.5]) $(p=0.117)$. Similarly, there was no significant difference between male $(n=33$; $57.9 \%)$ and female $(n=24 ; 42.1 \%)$ CE cases $(p=0.373)$. The majority ( 42 of $57 ; 74 \%$ ) of positive subjects were expatriates and the number of Syrian CE cases $(n=10$; $17.5 \%)$ was significantly higher in comparison to other nationalities $(p<0.001)$. The seropositivity (from 11 in 2010 to 1 in 2017) and the estimated incidence rate per 100,000 persons (from 0.31 to 0.025 ) of CE declined progressively from 2010 to 2,017 but showed a slight increase in 2018. However, there was no significant difference in annual ( $p=0.530)$ (Fig. 1$)$ or monthly occurrence $(p=$ 0.133) (Fig. 2) of CE cases in Kuwait. Similarly, there was no significant difference in the number of referrals from different hospitals/locations within Kuwait $(p=0.610)$ (Fig. 3).

\section{Discussion}

Kuwait has a large expatriate population mainly from south/southeast Asian and North African countries, many of which are endemic for parasitic and other infectious diseases [2-5]. In this retrospective study, the incidence of 2 parasitic diseases; schistosomiasis and CE was documented in Kuwait, based on serological data for subjects referred to PRL with a presumptive clinical diagnosis of schistosomiasis (186 of 997, 19.0\%) and hydatidosis (57 of 991, 5.8\%) from January 2010 to December 2018.

Schistosomiasis in the WHO EMR countries including Kuwait and other GCC countries is primarily seen in returning travelers from endemic African countries; particularly Egypt, whose nationals constitute a major expatriate population in these countries. In this study, the seropositivity and the estimated incidence rate per 100,000 persons of schistosomiasis significantly and progressively declined (from 50 in 2010 to 3 in 2018 and from 1.4 in 2010 to 0.07 in 2018 , respectively) during the study period $(p<0.001)$. Our data are consistent with a decreasing trend in the global burden of schistosomiasis, which has also declined from an estimated 3.3 million DALYs in 2010 to 1.496 million DALYs in 2016 [7, 11]. A study conducted in Najran, Saudi Arabia, reported a seropositivity rate of $20 \%$ and, similar to our study, positivity was high- 
er among male $(p<0.005)$ non-Saudi residents $(p<0.001)$ aged $20-40$ years $(p<0.001)$ [12]. Schistosomiasis prevalence has also declined in Oman over the years, from about $10 \%$ in $2003-0 \%$ in 2011 due to successful implementation of WHO strategic plan 2012-2020 to eradicate schistosomiasis via preventive chemotherapy, snail control, environmental management, health education, access to safe water, and sanitation [13]. Schistosomiasis cases have also declined among Egyptians, which constituted the major expatriate group testing positive for schistosomiasis in Kuwait. The prevalence of S. mansoni in the Nile Delta declined from $14.8 \%$ in $1993-2.7 \%$ in 2002 with a further decline to $1.5 \%$ in 2006 [13]. A recent study also reported a decline in the rate of heavy intensity $S$. haematobium infections among school children in Upper Egypt districts [14]. On the contrary, the disease prevalence has shown an increasing trend among inhabitants of fragile and conflict-affected areas (e.g., civil unrest in Burundi or war in Yemen and East/South Darfur), likely due to the influx of refugees, reduced adherence to treatment, etc. $[7,8,15]$. These observations suggest that elimination of schistosomiasis requires multifactorial actions including strict compliance of chemotherapy, knowledge about the disease and self-protection, and proper disposal of human excreta to avoid reinfection.

Although there was no age difference between schistosomiasis cases and noninfected subjects $(p=0.610)$, the majority of schistosomiasis cases were males $(n=169$; $90.9 \%)$ compared to females, and the difference was statistically significant $(p=0.043)$. Our data are consistent with previous reports from Egypt and other African countries describing higher prevalence of schistosomiasis among male individuals, who were 2.9 times more likely to be infected than females and accounted for $72 \%$ of heavy intensity infections in Upper Egypt districts [14$16]$.

All schistosomiasis cases in Kuwait were non-Kuwaiti expatriate residents and nearly all (129 of 130) infected patients for whom nationality status was known were Egyptians (the nationality of 56 non-Kuwaiti residents was not available). Egyptians constitute a major ethnic group among expatriates in Kuwait and are the most dominant nationality from countries endemic for schistosomiasis. It is probable that the majority of non-Kuwaiti expatriates whose nationality was not known may have also originated from Egypt.

As observed for schistosomiasis, the seropositivity (from 11 in 2010 to 1 in 2017) and the estimated incidence rate per 100,000 persons (from 0.31 to 0.025 ) of CE also declined progressively from 2010 to 2017 but showed a slight increase in 2018. In humans, CE is essentially a chronic process of slow cyst growth and depending on the site of infection, the disease may not manifest until many years after infection. Thus, many hydatid cysts are only discovered accidently during routine investigations for other conditions either by imaging and/or clinically during surgery, while others are found on autopsy. Based on these criteria, the CE seroprevalence data reported in this study are an underestimation of the true incidence as many CE cases in Kuwait were not referred for serological investigation. This is also supported by data reported previously from Kuwait showing an estimated incidence of $1.65 \mathrm{CE}$ cases per 100,000 persons based on a 6-year review of surgical records from District General Hospitals in Kuwait from 1980-1985 [17].

The overall prevalence of CE in the Arabian Peninsula has not been sufficiently investigated in recent years. One study reported a relatively low CE incidence $(0.6 \%)$ in UAE [18], while a higher prevalence of CE was reported from Yemen, where 66 cases were detected in a 6-month survey [19]. A more recent systematic review and metaanalysis from the Middle Eastern countries has also reported CE seroprevalence rates of 7.4 and $10.7 \%$ in the general population and in high-risk individuals, respectively [20]. Similarly, a systematic review and meta-analysis had reported higher pooled prevalence of CE and alveolar echinococcosis in Iran at 5 and 2\%, respectively [21]. In addition, it also detected a significant association of CE with keeping dogs as pets or contact with dogs $(p=$ 0.022 ) as well as consumption of raw or undercooked vegetables $(p<0.001)$ [22].

The seroprevalence of CE in our study was similar in younger versus older subjects and male $(n=33 ; 3.3 \%)$ versus female $(n=24 ; 2.4 \%)$ patients $(p>0.05)$. Although similar findings were reported from a high-risk area in Jordan [23], a recent systematic review and meta-analysis of 37 studies from Iran showed higher prevalence of CE in patients younger than 40 years of age [21]. A study from Iraq has shown that CE affected more females (61.2\%) than males (38.8\%) and more cases were reported in the age-group of 21-30 years than in other age-groups [24]. These contrasting results could reflect differences in exposure of positive cases to known risk factors among different populations in different settings.

The majority of CE cases in Kuwait were among nonKuwaiti expatriate residents (42 of 57; 73.7\%) compared with Kuwaiti nationals $(n=15)(p<0.05)$. Although the nationality of half ( 21 of $42,50 \%$ ) of all CE cases among expatriates was not known, the largest ethnic group among expatriate $\mathrm{CE}$ cases whose nationality was known 
were Syrian nationals $(n=10)$. Prolonged internal conflict in Syria, a predominantly rural country rich in agriculture and livestock farming, has adversely affected the preventive health services causing a major increase in parasitic and other tropical infections including CE [25]. Recent studies from adjoining and some European countries sheltering large number of Syrian refugees have also reported several cases of CE in asymptomatic and undiagnosed immigrant Syrian populations [26]. A recent field study carried out in Al-Mafraq City in northeastern Jordan, which hosts the largest number of Syrian refugees due to its proximity to the Syrian-Jordanian border, reported that 21 of 512 (4.1\%) participants were seropositive for $\mathrm{CE}$ and associated the risk of infection with slaughtering of sheep inside shelters instead of abattoirs (a common practice in rural/sub-urban Syria) and the presence of stray dogs [23].

Surprisingly, CE was also detected in 15 Kuwaiti nationals. However, the demographic data and the contact history of positive cases with livestock animals/stray dogs were not available due to the retrospective nature of the study. Nevertheless, common animal husbandry practices and other environmental similarities in the GCC region suggest that livestock infection rates are sufficiently high to maintain the life cycle of the parasite, and the consequent endemicity of human CE in the Middle East [27]. Camels, sheep, goats, and cattle are all significant intermediate hosts which are routinely raised in livestock farms in rural areas in many Middle Eastern countries. Although data on the E. granulosus infection of livestock animals in Kuwait are lacking, a number of studies in the region have reported varying infection rates among livestock animals (sheep, camels, and goats). Two recent studies have reported hydatidosis in $0.23 \%$ of 38,302 slaughtered goats in Makkah area [27] and 1.06\% hydatidosis in 12,569 slaughtered sheep in Riyadh [28], Saudi Arabia. Even higher infection rates have been reported in livestock animals in Iran [29]. Infection of sheep and camels by E. granulosus has also been detected in Oman [30]. Dogs, the definitive host of E. granulosus, also play an important role in the transmission of CE infection. Unfortunately, no data are available on the E. granulosus infection rate among stray dogs in Kuwait.

\section{Conclusion}

This retrospective study showed prevalence rates of schistosomiasis and CE as 19.0 and 5.8\%, respectively, in Kuwait. All schistosomiasis cases and a significant majority (73.7\%) of CE cases were seen among expatriates. There was a significant decrease in the number of both schistosomiasis and CE during the study period (20102018), which is attributed to the preventive health practices in Kuwait and successful implementation of WHO strategies to control and prevent schistosomiasis and CE in endemic countries.

\section{Statement of Ethics}

Due to the retrospective nature of this study, ethical clearance was not required as the extracted data did not include any personal information and the identity of subjects remained anonymous.

\section{Conflict of Interest Statement}

The authors declare no conflicts of interest.

\section{Funding Sources}

This study was funded by the College of Graduate Studies and Research Sector, Kuwait University (Project number YM 06/14).

\section{References}

1 Statistical Reports. The public authority for civil information. 2019. Available from: http: //stat.paci.gov.kw/stat/Default/aspx.

2 Herricks JR, Hotez PJ, Wanga V, Coffeng LE, Haagsma JA, Basáñez MG, et al. The global burden of disease study 2013: what does it mean for the NTDs? PLoS Negl Trop Dis. 2017;11(8):e0005424.

3 Al-Awadhi M, Iqbal J, Ahmad S. Cysticercosis, a potential public health concern in Kuwait: a new diagnostic method to screen Taenia solium taeniasis carriers in the expatriate population. Med Princ Pract. 2020;29:347-53.
4 Iqbal J, Al-Awadhi M, Ahmad S. Decreasing trend of imported malaria cases but increasing influx of mixed P. falciparum and P. vivax infections in malaria-free Kuwait. PLoS One. 2020;15(12):e0243617.

5 Mokaddas E, Ahmad S, Samir I. Secular trends in susceptibility patterns of Mycobacterium tuberculosis isolates in Kuwait, 19962005. Int J Tuberc Lung Dis. 2008;12:319-25.

6 Echinococcosis. World Health Organization. 2020. Available from: https://www.who.int/ news-room/fact-sheets/detail/echinococcosis.
7 Schistosomiasis. World Health Organization. 2020. Available from: https://www.who.int/ news-room/fact-sheets/detail/schistosomiasis.

8 Schistosomiasis. Chapter 4: 2020 Yellow Book, Travelers' Health, CDC. www.nc.cdc. gov. 2020. Available from: https://wwwnc. cdc.gov/travel/yellowbook/2018/infectiousdiseases-related-to-travel/schistosomiasis.

9 Koshy A, Gopalakrishnan G, Al-Mufti S, Hira PR, Al-Awadi KA, Al-Nakib B. Urinary schistosomiasis associated with hepatitis $\mathrm{C}$ virus infection. J Urol. 1995;153(3 Pt 1):698-700. 
10 Borhani M, Fathi S, Lahmar S, Ahmed H, Abdulhameed MF, Fasihi Harandi M. Cystic echinococcosis in the Eastern Mediterranean region: neglected and prevailing! PLoS Negl Trop Dis. 2020;14:e0008114.

11 Murray CJ, Vos T, Lozano R, Naghavi M, Flaxman AD, Michaud C, et al. Disability-adjusted life years (DALYs) for 291 diseases and injuries in 21 regions, 1990-2010: a systematic analysis for the Global burden of disease study 2010. Lancet. 2012;380:2197-223.

12 El-Shahawy IS, Khalil MI, Adail AS. Some epidemiological and serological studies on schistosomiasis in Najran area, Saudi Arabia. Helminthologia. 2016;53(2):172-9.

13 World Health Organization. Report on the intercountry meeting on strategies to eliminate schistosomiasis from the Eastern Mediterranean Region, Muscat, Oman, 6-8 November 2007. Apps.who.int. 2020. Available from:https://apps.who.int/iris/handle/10665/ 115981.

14 Haggag AA, Casacuberta Partal M, Rabiee A, Abd Elaziz KM, Campbell CH, Colley DG, et al. Multiple praziquantel treatments of Schistosoma mansoni egg-negative, CCA-positive schoolchildren in a very low endemic setting in Egypt do not consistently alter CCA results. Am J Trop Med Hyg. 2019;100(6):1507-11.

15 Cha S, Elhag MS, Lee YH, Cho DS, Ismai HAHA, Hong ST. Epidemiological findings and policy implications from the nationwide schistosomiasis and intestinal helminthiasis survey in Sudan. Parasit Vectors. 2019;12(1): 429.
16 Haggag AA, Rabiee A, Abd Elaziz KM, Gabrielli AF, Abdelhai R, Hashish A, et al. Elimination of schistosomiasis haematobia as a public health problem in five governorates in Upper Egypt. Acta Trop. 2018;188:9-15.

17 Humayun MS, Al-Manee MS. Hydatid cysts of the spleen. J Kuwait Med Assoc. 1987;21: 124-7.

18 Sultan DM, Sadjjadi SM, AbdRaboh NR. Serological evidence of Echinococcosis in municipality workers in United Arab Emirates. Res J Parasitol. 2016;11(3):33-40.

19 Alghoury A, El-Hamshary E, Azazy A, Hussein E, Rayan HZ. Hydatid disease in Yemeni patients attending public and private hospitals in Sana'a City, Yemen. Oman Med J. 2010; 25:88-90.

20 Galeh TM, Spotin A, Mahami-Oskouei M, Carmena D, Rahimi MT, Barac A, et al. The seroprevalence rate and population genetic structure of human cystic echinococcosis in the Middle East: a systematic review and meta-analysis. Int J Surg. 2018;51:39-48.

21 Mahmoudi S, Mamishi S, Banar M, Pourakbari B, Keshavarz H. Epidemiology of echinococcosis in Iran: a systematic review and meta-analysis. BMC Infect Dis. 2019;19(1):929.

22 Sakhaei G, Khademvatan S, HazratiTappeh K, Masudi S, Feizollahzadeh S, Aminpour A, et al. Sero-epidemiology of hydatidosis among general population of Jolfa county, Northwestern Iran using IHA, ELISA and Western Blot (2017-2018). Infect Disord Drug Targets. 2020 May 16.

23 Himsawi N, Hijjawi N, Al-Radaideh A, AlTamimi M. Seroprevalence of cystic echinococcosis in a high-risk area (Al-Mafraq Governorate) in Jordan, using indirect hemagglutination test. Parasite Epidemiol Control. 2019;5:e0104.
24 Abdulhameed MF, Habib I, Al-Azizz SA, Robertson I. A retrospective study of human cystic echinococcosis in Basrah province, Iraq. Acta Trop. 2018;178:130-3.

25 Centers for Disease Control and Prevention. Parasitic infections: Syrian refugee health profile. CDC; 2020. Available from: https:// www.cdc.gov/immigrantrefugeehealth/profiles/syrian/health-information/parasitic-infections/index.html.

26 Echinococcosis. Ecdc.europa.eu. 2020. Available from: https://www.ecdc.europa.eu/sites/ default/files/documents/AER_for_2016echinococcosis.pdf.

27 Alsulami M. Prevalence and histopathological study on cystic hydatidosis in heart and spleen of goat slaughtered at Makkah, Saudi Arabia. Ann Parasitol. 2019;65:225-36.

28 Almalki E, Al-Quarishy S, Abdel-Baki AS. Assessment of prevalence of hydatidosis in slaughtered Sawakny sheep in Riyadh city, Saudi Arabia. Saudi J Biol Sci. 2017;24:15347.

29 Ghasemian O, Hoseini G, Soleimani M, Mahmoudi R, Kaboudari A. The prevalence study of hydatid cyst in domesticated slaughtered animals in industrial abattoirs in Iran. J Bacteriol Mycol Open Access. 2018;6:96-100

30 AlKitani FA, Baqir S, Mansoor MK, AlRiyami S, Hussain MH, Roberts D. Genetic survey of cystic echinococcosis in farm animals in Oman. Trop Anim Health Prod. 2020;52(1): $331-7$.
Imported Schistosomiasis and CE in Kuwait
Med Princ Pract 2021;30:138-145

DOI: $10.1159 / 000514873$ 